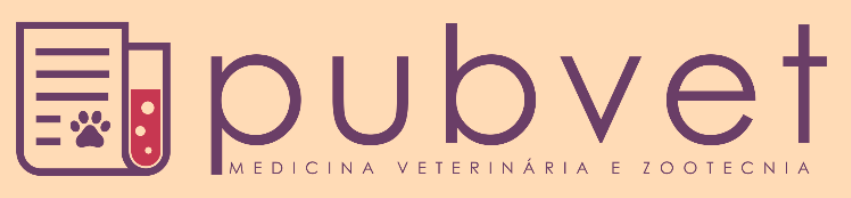

https://doi.org/10.31533/pubvet.v14n7a614.1-9

\title{
Casuística de micoses em pequenos animais atendidos em Hospital Veterinário Universitário do Ceará: estudo retrospectivo
}

\author{
Rosane de Oliveira Cruz $^{1 *}$, Adriana de Queiroz Pinheiro ${ }^{2} \bullet$, Barbara Wilka Leal Silva ${ }^{3}$, $^{\circ}$ \\ Géssica dos Santos Araújo ${ }^{4}{ }^{\circ}$, Lorena Mayana Beserra de Oliveira ${ }^{50}$ \\ ${ }^{I}$ Médica veterinária formada pela Universidade Estadual do Ceará, Faculdade de Veterinária. Fortaleza - CE Brasil. \\ ${ }^{2}$ Professora da Universidade Estadual do Ceará, Faculdade de Veterinária. Fortaleza - CE Brasil \\ ${ }^{3}$ Médica Veterinária Residente - Universidade Federal Rural da Amazônia, Faculdade de Veterinária. Belém - PA Brasil. \\ ${ }^{4}$ Pesquisadora - Universidade Federal do Ceará, Centro de Estudos em Micologia Médica. Fortaleza - CE Brasil. \\ ${ }^{5}$ Professora do Programa de Pós-graduação em Ciências Veterinárias - PPGCV - Universidade Estadual do Ceará - UECE. Fortaleza - CE Brasil \\ * Autora para correspondência, E-mail: rosane_oliveiracruz@hotmail.com
}

Resumo. A utilização crescente de terapias imunossupressivas e o surgimento de infecções retrovirais, tanto em humanos como animais, foram decisivas para a expansão de doenças oportunistas, em grande parte fúngicas. Tendo em vista esta realidade, desde 2013 o Laboratório de Microbiologia Veterinária (LAMICRO) da Faculdade de Veterinária da Universidade Estadual do Ceará desenvolve atividades de pesquisa e extensão fornecendo diagnóstico micológico para amostras oriundas de pequenos animais atendidos na Unidade Hospitalar Veterinária e posteriormente no Hospital Veterinário Sylvio Barbosa Cardoso. Assim, objetivouse documentar a casuística e os diagnósticos realizados pelo LAMICRO em um período de 5 anos, enfatizando a importância do diagnóstico micológico e contribuindo para o estabelecimento do perfil epidemiológico na região. Para tanto, foi realizado uma avaliação retrospectiva das fichas contendo os dados dos animais, os sinais clínicos, a suspeita principal e os demais exames solicitados. Entre julho de 2013 a dezembro de 2018 foram recebidas 2.431 amostras, dentre estas 543 foram positivas para algum tipo de micose de importância veterinária. Os casos de micoses, diagnosticadas no período de 5 anos, acometeram preponderantemente caninos entre 2 e 10 anos de idade e felinos com até 2 anos de idade; foram mais frequentes em cães e gatos sem raça definida, manifestaram-se em ambas as espécies, por lesões alopécicas, difusas e crostosas; topograficamente, observaram-se lesões assestadas em ambas as espécies nas regiões cefálica, torácica, de membros, cervical e abdominal; tiveram como agentes etiológicos mais comuns Malassezia pachydermatis e Microsporum canis. O conhecimento da epidemiologia das micoses em animais de estimação, principalmente dos dermatófitos, é importante para diminuir a disseminação de infecção por fungos zoofilicos para humanos e auxiliar na obtenção de medidas profiláticas na disseminação da doença entre animais. $\mathrm{O}$ exame direto, utilizado no presente estudo, é uma importante forma de diagnóstico e pode auxiliar o clínico reduzindo o tempo necessário para o início do tratamento, no entanto ainda é pouco sensível, dessa forma faz se necessário o uso da cultura fúngica para obter melhores resultados; ambos, associados à clínica do animal, fornecem um diagnóstico mais preciso para que a terapêutica estabelecida seja eficaz para um melhor tratamento e prognóstico dos casos clínicos.

Palavras chave: cães, epidemiologia, fungos, gatos

\section{Casuistry of mycoses in small animals attended at the University Veterinary Hospital of Ceará: restrospective study}

Abstract. The increased use of immunosuppressive therapies and the emergence of retroviral infections, both in humans and animals, have been decisive for the expansion of opportunistic, largely fungal diseases. In view of this reality, since 2013 the 
Laboratório de Microbiologia Veterinária (LAMICRO) of the Faculty of Veterinary of the State University of Ceará develops research and extension activities providing mycological diagnosis for samples from small animals attended at the Veterinary Hospital Unit and later at the Veterinary Hospital Sylvio Barbosa Cardoso. Thus, the objective was to document the casuistry and the diagnoses made by LAMICRO in a period of 5 years, emphasizing the importance of the mycological diagnosis and contributing to the establishment of the epidemiological profile in the region. For this, a retrospective evaluation of the records containing the data of the animals, the clinical signs, the main suspicion and the other exams requested was performed. Between July 2013 and December 2018, 2.431 samples were received, among these 543 were positive for some type of mycosis of veterinary importance. The cases of mycosis, diagnosed in the period of 5 years, affected predominantly canines between 2 and 10 years of age and cats up to 2 years of age; they were more frequent in dogs and cats without a defined breed, manifested in both species, by allopecic, diffuse and crusted lesions; topographically, lesions were observed in both species in the cephalic, thoracic, limb, cervical and abdominal regions; they had as more common etiological agents, in both species, Malassezia pachydermatis and Microsporum canis. Knowledge of the epidemiology of mycoses in pets, especially dermatophytes, is important to reduce the spread of infection by zoofilic fungi to humans and help to obtain prophylactic measures in the spread of the disease among animals. The direct examination, used in the present study, is an important form of diagnosis and can help the clinician reducing the time necessary for the beginning of the treatment, however it is still little sensitive, this way it makes if necessary the use of the fungal culture to obtain better results; both, associated to the animal clinic, provide a more precise diagnosis so that the established therapy is effective for a better treatment and prognosis of the clinical cases.

Keywords: cats, dogs, epidemiology, yeast

\section{Casuística de micosis en pequeños animales atendidos en el Hospital Veterinario Universitario de Ceará: estudio retrospectivo}

Resumen. El creciente uso de terapias inmunosupresoras y la aparición de infecciones retrovirales, tanto en humanos como en animales, han sido decisivos para la expansión de enfermedades oportunistas, en gran parte fúngicas. Ante esta realidad, desde 2013 el Laboratorio de Microbiología Veterinaria (LAMICRO) de la Facultad de Veterinaria de la Universidad Estatal de Ceará desarrolla actividades de investigación y extensión que permiten el diagnóstico micológico de muestras de pequeños animales atendidas en la Unidad Hospitalaria Veterinaria y posteriormente en el Hospital Veterinario Sylvio Barbosa Cardoso. Así, el objetivo fue documentar la casuística y los diagnósticos realizados por LAMICRO en un período de 5 años, destacando la importancia del diagnóstico micológico y contribuyendo al establecimiento del perfil epidemiológico en la región. Para ello, se realizó una evaluación retrospectiva de los registros que contenían los datos de los animales, los signos clínicos, la principal sospecha y los demás exámenes solicitados. Entre julio de 2013 y diciembre de 2018 se recibieron 2.431 muestras, de las cuales 543 dieron positivo en algún tipo de micosis de importancia veterinaria. Los casos de micosis, diagnosticados en el período de 5 años, afectaban predominantemente a caninos de entre 2 y 10 años de edad y a gatos de hasta 2 años de edad; eran más frecuentes en perros y gatos sin raza definida, manifestándose en ambas especies, por lesiones alopécicas, difusas y costrosas; topográficamente, se observaron lesiones en ambas especies en las regiones cefálica, torácica, de las extremidades, cervical y abdominal; tenían como agentes etiológicos más comunes, en ambas especies, Malassezia pachydermatis y Microsporum canis. El conocimiento de la epidemiología de las micosis en los animales domésticos, especialmente los dermatofitos, es importante para reducir la propagación de la infección por hongos zoofílicos a los seres humanos y ayudar a obtener medidas profilácticas en la difusión de la enfermedad entre los 
animales. El examen directo, utilizado en el presente estudio, es una forma importante de diagnóstico y puede ayudar al clínico a reducir el tiempo necesario para el inicio del tratamiento, sin embargo todavía es poco sensible, de esta manera se hace si es necesario el uso del cultivo de hongos para obtener mejores resultados; ambos, asociados a la clínica animal, proporcionan un diagnóstico más preciso para que la terapia establecida sea efectiva para un mejor tratamiento y pronóstico de los casos clínicos.

Palabras clave: epidemiologia, gatos, hongos, perros

\section{Introdução}

As dermatomicoses são infecções cutâneas cosmopolitas com maior prevalência em regiões de clima quente e úmido (Chaves, 2007). Tanto na medicina humana como veterinária, são reconhecidas como um dos principais motivos de visitas às clínicas. Dados de Hospitais Veterinários de Instituições de Ensino apontam índices variados na etiologia fúngica das infecções de pele de cães e gatos. Essas infecções coexistem com o homem em centros urbanos e podem desempenhar importante papel na epidemiologia das micoses, seja como seu vetor direto ou intermediário, seja como sentinela do fungo patogênico no ambiente (Paterson, 2010).

No Brasil, ainda são poucos os estudos abrangentes abordando prevalência das doenças causadas por fungos em animais nas diferentes regiões do país, sobretudo naqueles centros onde as características climáticas e socioeconômicas são mais favoráveis a incidência de doenças fúngicas. Nesse contexto, a investigação micológica pode contribuir de forma relevante para o conhecimento epidemiológico da doença, da distribuição geográfica dos fungos patogênicos, bem como da relação entre a ocorrência dos fungos em animais e a população. Valendo ainda ressaltar que na atualidade, a maior parte das publicações são mais voltadas a levantamentos fragmentados e relatos de casos específicos. Ademais, com o passar do tempo, podem haver alterações no perfil epidemiológico das micoses em cada região. Desse modo, estudos epidemiológicos devem ser realizados periodicamente, a fim de revalidar os dados presentes na literatura acerca dessas infecções.

Assim, objetivou-se documentar a casuística e os diagnósticos de doenças fúngicas realizados pelo LAMICRO em um período de 5 anos, enfatizando a importância do diagnóstico micológico e contribuindo para o estabelecimento do perfil epidemiológico na região.

\section{Material e métodos}

\section{Animais}

Espécimes clínicos foram obtidos de cães e gatos apresentando sinais clínicos e lesões dermatológicas conforme parecer do Médico Veterinário. Os animais foram atendidos no período de 2013 a 2017 na Unidade Hospitalar Veterinária e no período de 2017 a 2018, após expansão dos serviços prestados pela Faculdade de Veterinária, no Hospital Veterinário Sylvio Barbosa Cardoso, ambos na Universidade Estadual do Ceará.

\section{Espécimes clínicos}

As amostras (raspados de pele, swabs, punções, lavados, fragmentos teciduais, debris celulares) enviadas ao laboratório de Microbiologia Veterinária, acompanhadas de ficha de solicitação devidamente preenchidas e apresentando dados sobre o animal (espécie, raça, idade), uso de antimicrobianos, demais sintomas clínicos, suspeita principal, informações sobre os demais exames solicitados (bacteriológicos, hematológicos, bioquímicos histopatológicos), hipótese diagnóstica foram processadas e as fichas analisadas. $\mathrm{Na}$ análise retrospectiva, os dados da rotina micológica do Laboratório de Microbiologia da FAVET, referentes ao período de julho de 2013 a dezembro de 2018, foram analisados pelo estudo das fichas do referido período.

\section{Processamento de amostras}

Exame direto: No laboratório, as amostras de pêlos e escamas foram processadas, após a cultura, em local asséptico com auxílio do bico de Bunsen, e em seguida, foram depositadas sobre lâminas, 
clarificadas em solução de hidróxido de potássio (KOH 10\%) e cobertas com uma lamínula. O conjunto pode ser aquecido suavemente por alguns segundos em bico de Bunsen para acelerar o clareamento. Após 10 a 15 minutos foi feita a análise microscópica da lâmina para visualização de possíveis estruturas fúngicas como os artroconídios. Essas estruturas podem ser confundidas com artefatos como filamentos e fibras de algodão, ou ainda com formações de cristal de $\mathrm{KOH}$ em preparações superaquecidas ou ressecadas. Em amostras de pelo, o tamanho do artroconído e sua posição fora ou dentro do pelo são utilizados para auxilio a identificação da espécie, caracterizando em parasitismo endotrix (dentro) ou ectotrix (fora).

O processamento de swabes de mucosas, lesões supurativas de pele e/ ou de aspirados de nódulos; fragmentos teciduais e lavados foram feitos por meio de citologia do esfregaço da secreção, corado pelo método de Gram e pelo Panótico Rápido.

Cultura e identificação: O diagnóstico definitivo de espécies patogênicas de fungos foi feito através de cultura fúngica. Foi realizada a semeadura das amostras em três meios: Agar Sabouraud Dextrose, Agar Saboraud com Cloranfenicol e Agar Saboraud com Ciclohexamida, incubada a temperatura ambiente (em torno de $25^{\circ} \mathrm{C}$ ) por até 21 dias. As colônias obtidas foram analisadas por suas características macro e microscópicas. Diante do crescimento da colônia procede-se ao exame macroscópico (cor, textura) e microscópico. O exame microscópico foi realizado através de preparações com lactofenol azul de algodão para identificação das espécies de fungos filamentosos, com base nas características peculiares das estruturas de frutificação. Caso houvesse necessidade, era realizado o microcultivo de amostras nas quais a identificação através da análise microscópica não foi possível. O microcultivo em ágar batata é usado para proporcionar um estudo detalhado das diferentes estruturas fúngicas, bem como a disposição destas ao longo das hifas. A preparação dos blocos é feita mediante o corte com bisturi de uma película de aproximadamente $0,4 \mathrm{~mm}$ de profundidade de ágar-batata em uma placa de Petri. Os blocos quadriculares são transferidos para lâminas de microscopia estéreis e então inoculados nos quatro lados com um pequeno fragmento da colônia do isolamento primário. Em seguida, são cobertos com lamínula estéril e incubados em uma câmara úmida estéril, à temperatura ambiente, por um período de aproximadamente quinze a vinte dias, quando houver crescimento adequado, a lamínula, com o micélio aderido, é removida do bloco de ágar e montada sobre uma lâmina de microscopia contendo o corante lactofenol azul-algodão.

\section{Análise dos resultados}

Os resultados obtidos dos exames micológicos foram relacionados à busca ativa de dados epidemiológicos, clínicos, bioquímicos, sorológicos, histo e citopatológicos de cada animal.

\section{Análise estatística}

Utilizou-se o teste de duas proporções, com aproximação pela distribuição normal de probabilidades, a fim de verificar a existência de diferença estatisticamente significante entre os resultados obtidos. O nível de significância (valor crítico) adotado foi de 5\% para as análises realizadas.

\section{Resultados e discussão}

Durante o intervalo do estudo foram recebidas e processadas um total de 2.431 amostras, dentre estas, $543(22,3 \%)$ foram considerados positivas para dermatopatia de origem fúngica. Sendo ainda dentre estas 543, 194 amostras correspondentes a algum agente causador de dermatofitose, o que equivale a $8,0 \%$ do total de amostras recebidas e a 26,6 \% do total de amostras positivas, enfatizando a importância do conhecimento da epidemiologia dos dermatófitos uma vez que trata se de uma importante zoonose (Tabela 1). Entretanto é importante salientar que nem todas as amostras recebidas eram suspeitas de dermatofitose, uma vez que também eram enviadas amostras com suspeitas de outras dermatomicoses e ácaros, o que pode ter subestimado o percentual encontrado. Durante o intervalo de estudo foram diagnosticadas 185 dermatopatias de origem parasitária dentre as 2.431 amostras recebidas, o que corresponde a $7,6 \%$ do total de amostras recebidas e a $25,4 \%$ do total de amostras positivas. 
Para algumas amostras a cultura fúngica não foi solicitada pelo médico veterinário, sendo o diagnóstico positivo obtido somente através do exame direito e dessa forma impossibilitando o diagnóstico da espécie envolvida, entretanto nas amostras que foram visualizados artroconídios no exame direto, mesmo na ausência de cultura fúngica foram agrupadas na categoria dos dermatófitos. No presente estudo, na categoria dos dermatófitos estão agrupados os gêneros Microsporum e Trichophyton. Na categoria leveduras patogênicas cuja amostra clínica foi o raspado encontram-se espécies dos gêneros Malassezia e Candida; nas amostras clínicas de aspirado cutâneo, dentre os fungos dimórficos estão os patógenos do gênero Histoplasma; já as leveduras patogênicas são constituídas por fungos do gênero Cryptococcus.

Tabela 1. Amostras clinicas e patógenos identificados recebidos no período entre julho de 2013 a dezembro de 2018.

\begin{tabular}{llcc}
\hline Amostra clínica & Patógeno & $\mathrm{n}$ & $\%$ \\
\hline \multirow{2}{*}{ Raspado de pele } & Dermatófitos & 194 & $26,6 \%$ \\
& Leveduras patogênicas & 298 & $40,9 \%$ \\
& Ácaros & 185 & $25,4 \%$ \\
\hline Swab auricular & Malassezia pachydermatis & 36 & $04,9 \%$ \\
\hline \multirow{2}{*}{ Aspirado cutâneo } & Fungos dimórficos & 07 & $01,0 \%$ \\
& Leveduras patogênicas & 08 & $01,1 \%$ \\
\hline Total & & 728 & $100 \%$ \\
\hline
\end{tabular}

A espécie animal acometida predominantemente foram os cães, responsáveis por $81,4 \%$ do número total de animais acometidos por alguma micose. Os gatos foram responsáveis por $18,6 \%$ do número total. Para os dermatófitos especificamente, foi observado uma prevalência de $62,9 \%$ para cães e 37,1\% para gatos (Tabela 2). Segundo Brilhante et al. (2003), o percentual de animais positivos em relação ao número total de animais suspeitos de dermatofitose varia de $7 \%$ a $40 \%$ em cães e de 9\% a 46\% em gatos variando de acordo com a região, o que difere dos valores encontrados para os cães no presente estudo, possivelmente devido a uma predominância de atendimentos da espécie canina o que contribuiu com o aumento da sua prevalência; mas corrobora os achados para os gatos. No entanto, a ocorrência das dermatofitoses varia de acordo com o clima, temperatura, umidade relativa e níveis de precipitação pluviométrica, fatores que divergem entre as regiões geográficas, explicando possíveis contrastes entre as pesquisas (Silva et al., 2011).

Tabela 2. Relação entre os patógenos fúngicos identificados e espécie animal acometida.

\begin{tabular}{llccccc}
\hline Amostra clínica & Patógeno & Canina & $\%$ & Felina & $\%$ & Total \\
\hline \multirow{2}{*}{ Raspado de pele } & Dermatófitos & 122 & $62,9 \%$ & 72 & $37,1 \%$ & 194 \\
& Leveduras patogênicas & 285 & $95,6 \%$ & 13 & $04,4 \%$ & 298 \\
\hline Swab auricular & Malassezia pachydermatis & 31 & $86,1 \%$ & 05 & $12,9 \%$ & 36 \\
\hline \multirow{2}{*}{ Aspirado cutâneo } & Fungos dimórficos & 00 & $00 \%$ & 07 & $100 \%$ & 07 \\
& Leveduras patogênicas & 04 & $50 \%$ & 04 & $50 \%$ & 08 \\
\hline Total & & 442 & & 101 & & 543 \\
\hline
\end{tabular}

A faixa etária dos animais do estudo variou entre 3 meses a 14 anos, sendo para os cães a faixa etária mais prevalente com 46,2\% dos animais entre 2 e 10 anos e para os gatos houve a predominância de 46,5\% dos animais com até 2 anos de idade (Tabela 3). Cafarchia et al. (2004) e Cabanes et al. (1997) relataram maior ocorrência de infecções em animais com idade inferior a um ano de idade.

Tabela 3. Faixa etária dos animais do estudo.

\begin{tabular}{lcccc}
\hline Idade & Cães & $\%$ & Gatos & $\%$ \\
\hline Não informada & 38 & $8,6 \%$ & 33 & $32,7 \%$ \\
Até 2 anos & 175 & $39,6 \%$ & 47 & $46,5 \%$ \\
$>2-10$ anos & 204 & $46,2 \%$ & 19 & $18,8 \%$ \\
$>10$ anos & 25 & $5,6 \%$ & 02 & $02,0 \%$ \\
\hline Total & 442 & $100 \%$ & 101 & $100 \%$ \\
\hline
\end{tabular}


No presente estudo, entretanto, foi observado um predomínio de cães adultos e de gatos jovens. Segundo Balda et al. (2004) ocorre uma predominância de acometimento de animais jovens possivelmente devido à imaturidade do sistema imunológico desses animais tornando os mais susceptíveis a infecção. Além disso, por apresentarem deficiência de ácido linoleico que desempenha um papel fungistático e por desenvolver mudanças bioquímicas na pele ocorrendo durante a fase anágena de crescimento dos pelos (Lewis et al., 1991).

Tanto para cães quanto para gatos houve uma predominância de animais sem padrão racial definido; sendo possível observar o percentual de $51,7 \%$ para cães e $76,2 \%$ para gatos. Com relação ao padrão racial dos cães acometidos, os resultados do presente estudo difere do que foi encontrado nos trabalhos de Palumbo et al. (2010) que encontraram uma predominância de cães da raça Poodle; e dos trabalhos de Balda et al. (2004), Cafarchia et al. (2004) e Sparkes et al. (1993) que encontraram predomínio de cães acometidos da raça Yorkshire Terrier. Além disso, esses estudos apontam os animais de raça como mais susceptíveis por serem mais sensíveis a infecções em oposto a rusticidade e maior resistência dos animais sem padrão racial definido. Esse perfil encontrado no presente estudo ocorreu possivelmente devido ao perfil sócio econômico do público do Hospital Veterinário Universitário, o qual devido ao seu caráter social atende predominantemente a população com menor poder aquisitivo, a qual na sua maioria possui animais sem padrão racial definido. Entretanto seguido aos cães sem raça definida, foi observado um predomínio de infecção em cães da raça Poodle $(17,6 \%)$ como observado por Palumbo et al. (2010), seguido de Pinschers (7,6\%) e Yorkshires (7,1\%) (Tabela 4).

Tabela 4. Prevalência de raças caninas afetadas.

\begin{tabular}{lcclcc}
\hline Raça dos cães & $\mathrm{n}$ & $\%$ & Raça dos cães & $\mathrm{n}$ & $\%$ \\
\hline SRD & 203 & $51,7 \%$ & Akita & 3 & $0,8 \%$ \\
Poodle & 69 & $17,6 \%$ & Beagle & 3 & $0,8 \%$ \\
Pinscher & 30 & $7,6 \%$ & Boxer & 3 & $0,8 \%$ \\
Yorkshire Terrier & 28 & $7,1 \%$ & Dálmata & 3 & $0,8 \%$ \\
Pitbull & 18 & $4,6 \%$ & Labrador & 3 & $0,8 \%$ \\
Pastor Alemão & 12 & $3,1 \%$ & Weimaraner & 3 & $0,8 \%$ \\
Cocker Spaniel & 11 & $2,8 \%$ & BullDog & 2 & $0,5 \%$ \\
Dachshund & 7 & $1,8 \%$ & GoldenRetrivier & 2 & $0,5 \%$ \\
Shih tzu & 7 & $1,8 \%$ & Staffordshireterrier & 2 & $0,5 \%$ \\
Chow Chow & 6 & $1,5 \%$ & Fila & 1 & $0,3 \%$ \\
Teckel & 5 & $1,3 \%$ & FoxPaulistinha & 1 & $0,3 \%$ \\
Pug & 5 & $1,3 \%$ & Nãoinformado & 1 & $0,3 \%$ \\
Maltês & 4 & $1,0 \%$ & Sharpei & 1 & $0,3 \%$ \\
Rottweiler & 4 & $1,0 \%$ & WestHighland & 1 & $0,3 \%$ \\
Schnauzer & 4 & $1,0 \%$ & Subtotal & 29 & $7,5 \%$ \\
Subtotal & 413 & $92,5 \%$ & Total & 442 & $100 \%$ \\
\hline
\end{tabular}

Com relação aos gatos, a predominância da ausência de padrão racial corrobora com o estudo de Palumbo et al. (2010) e Balda et al. (2004), entretanto, entre os animais com padrão racial definido destacam se a raça Persa $(9,9 \%)$ (Tabela 5). Essa é citada em outros trabalhos como os de Stannard \& Pulley (1978) e Miller et al. (2013) como mais prevalente entre os gatos. Balda et al. (2004) apontam como hipótese, uma disfunção relacionada as células do sistema imune, o que pode tornar esses animais mais susceptíveis a infecção; e ainda que o comprimento do pelame possa influenciar. Segundo $\underline{\text { Sparkes et al. }}$ (1993), possivelmente, os pêlos alongados facilitam as condições ótimas de temperatura e umidade para que as estruturas fúngicas fiquem protegidas contra a dissecação, favorecendo assim a sua propagação.

Os tipos de lesões encontrados predominantemente em cães foram lesões alopécicas $(32,2 \%)$, difusas $(25,9 \%)$ e crostosas $(25,2 \%)$. Os gatos apresentaram os mesmos tipos de lesões: predominantemente alopecias $(37,3 \%)$, difusas $(20,6 \%)$ e circulares $(18,6 \%)$ (Tabela 6). Ambos concordam com estudos anteriores de Balda et al. (2004) e Neves et al. (2011). Tanto em cães quanto em gatos, diferentes tipos de lesões coexistem no mesmo animal. 
Tabela 5. Prevalência de raças felinas afetadas.

\begin{tabular}{lcc}
\hline Raça dos gatos & $\mathrm{n}$ & $\%$ \\
\hline SRD & 77 & $76,2 \%$ \\
Persa & 10 & $9,9 \%$ \\
Pelo curto & 09 & $8,9 \%$ \\
Siamês & 05 & $5,0 \%$ \\
Total & 101 & $100 \%$ \\
\hline
\end{tabular}

Quanto a localização topográfica das lesões, em cães foram predominantemente as regiões cefálica $(12,4 \%)$, lombar $(11,6 \%)$, torácica $(11,3 \%)$, membros torácicos $(11,3 \%)$, membros pélvicos $(11,2 \%)$, e cervical $(9,4 \%)$. Localizações similares foram acometidas nos gatos: as regiões cefálicas $(19,2 \%)$, caudal $(12,8 \%)$, cervical $(9,9 \%)$, lombar $(9,9 \%)$ e abdominal $(9,9 \%)$ foram as mais predominantes (Tabela 7). Assim como os tipos de lesões, as localizações eram variadas e coexistiam no mesmo animal. Balda et al. (2004) apontam em seu trabalho a região cervical como a mais afetada em cães. Outros autores relatam que tanto em cães quanto em gatos, as regiões cefálica, membros e região caudal como as mais acometidas (Menges \& Georg, 1957).

Tabela 6. Prevalência de tipos lesionais nos animais acometidos.

\begin{tabular}{lcccc}
\hline Lesões & Canina & $\%$ & Felina & $\%$ \\
\hline Alopécicas & 138 & $32,2 \%$ & 38 & $37,3 \%$ \\
Difusa & 111 & $25,9 \%$ & 21 & $20,6 \%$ \\
Crostosas & 108 & $25,2 \%$ & 18 & $17,6 \%$ \\
Circular & 43 & $10,0 \%$ & 19 & $18,6 \%$ \\
Ulceradas & 09 & $2,1 \%$ & 02 & $2,0 \%$ \\
Nodulares & 07 & $1,6 \%$ & 02 & $2,0 \%$ \\
Purulentas & 13 & $3,0 \%$ & 02 & $2,0 \%$ \\
Total & 429 & $100,0 \%$ & 102 & $100,0 \%$ \\
\hline
\end{tabular}

O agente fúngico predominante em amostras oriundas de raspado de pele em cães foi a Malassezia pachydermatis (40,0\%), seguida de Microsporum canis (16,5\%), Trichophyton mentagrophytes (4,5\%), Microsporum gypseum (2,5\%), Trichophyton verrucosum (0,7\%), Trichophyton tonsurans $(0,2 \%)$ e Candida spp $(0,18 \%)$. Entre as amostras oriundas de punções e fragmentos teciduais houve uma predominância do agente Cryptococcus spp $(0,9 \%)$. Em gatos a espécie predominante foi Microsporum canis $(51,5 \%)$ seguida por Malassezia pachydermatis $(11,9 \%)$, Histoplasma spp $(6,9)$, Trichophyton mentagrophytes (5,9\%), Microsporum gypseum (5,0 \%) e Cryptococcus spp. $(4,0 \%)$ (Tabela 8).

Tabela 7. Prevalência de localização das lesões nos animais acometidos.

\begin{tabular}{lcccc}
\hline Localização & Canina & $\%$ & Felina & $\%$ \\
\hline Cefálica & 104 & $12,4 \%$ & 27 & $19,2 \%$ \\
Cervical & 79 & $9,4 \%$ & 14 & $9,9 \%$ \\
Torácica & 95 & $11,3 \%$ & 13 & $9,2 \%$ \\
Lombar & 97 & $11,6 \%$ & 14 & $9,9 \%$ \\
Sacral & 63 & $7,5 \%$ & 12 & $8,5 \%$ \\
Caudal & 64 & $7,6 \%$ & 18 & $12,8 \%$ \\
Peitoral & 72 & $8,6 \%$ & 09 & $6,4 \%$ \\
Abdominal & 77 & $9,2 \%$ & 14 & $9,9 \%$ \\
Membro torácico & 95 & $11,3 \%$ & 08 & $5,7 \%$ \\
Membro pélvico & 94 & $11,2 \%$ & 12 & $8,5 \%$ \\
Total & 840 & $100 \%$ & 141 & $100,00 \%$ \\
\hline
\end{tabular}

A literatura mostra predomínio da espécie Microsporum canis como agente prevalente, entretanto tais levantamentos se restringem aos gêneros dos dermatófitos, isso devido a sua alta prevalência dentre as dermatopatias fúngicas como também foi observado no presente trabalho. Assim, no tocante 
ao principal gênero de dermatófito, o presente estudo corrobora os dados dos estudos de Palumbo et al. (2010), Balda et al. (2004) e Cafarchia et al. (2004), mas inviabiliza a comparação com os demais agentes causadores de micoses superficiais como é o caso da Malassezia pachydermatis e de micoses sistêmicas como Cryptococcus spp. e Histoplasma spp.

Tabela 8. Prevalência de espécies fúngicas.

\begin{tabular}{lcccc}
\hline Espécie & Canina & $\%$ & Felina & $\%$ \\
\hline M. pachydermatis (ouvido) & 31 & $7,0 \%$ & 05 & $5,0 \%$ \\
M. pachydermatis (pele) & 177 & $40,0 \%$ & 12 & $11,9 \%$ \\
Candida & 01 & $0,2 \%$ & 00 & $0,0 \%$ \\
M. canis & 73 & $16,5 \%$ & 52 & $51,5 \%$ \\
M. gypseum & 11 & $2,5 \%$ & 05 & $5,0 \%$ \\
T. mentagrophytes & 20 & $4,5 \%$ & 06 & $5,9 \%$ \\
T. verrucosum & 03 & $0,7 \%$ & 00 & $0,0 \%$ \\
T. tonsurans & 01 & $0,2 \%$ & 00 & $0,0 \%$ \\
Histoplasma spp & 00 & $0,0 \%$ & 07 & $6,9 \%$ \\
Cryptococcus spp & 04 & $0,9 \%$ & 04 & $4,0 \%$ \\
* Artroconídios (direto) & 14 & $3,2 \%$ & 09 & $8,9 \%$ \\
* Leveduras (direto) & 107 & $24,2 \%$ & 01 & $1,0 \%$ \\
Total & 442 & $100,0 \%$ & 101 & $100,0 \%$ \\
\hline
\end{tabular}

*Diagnóstico realizado com base apenas no exame direto não sendo possível identificar a espécie.

No presente estudo, as espécies fúngicas foram em quase sua totalidade diagnosticadas através do exame direto associado a cultura micológica, conforme indicado por Moriello et al. (2017) que afirmam que o diagnóstico das dermatofitoses deve ser realizado por meio de um conjunto de testes diagnósticos complementares. Pode se notar que com relação as amostras oriundas de punções aspirativas e swabes de mucosas houve uma boa correlação entre o exame direto e a cultura variando entre 75 a 100\% de correlação ao longo dos anos, entretanto nas amostras oriundas do raspado de pele essa correlação foi baixa, variando de $1,5 \%$ a $50 \%$ ao longo dos anos avaliados. Este fato também foi observado por Coelho et al. (2008), constatando a baixa sensibilidade do exame direto. Este fato reafirma a necessidade de associação de exames complementares tais como a cultura fúngica, citologia, lâmpada de Wood ao histórico e exame físico do paciente como destacado por Moriello (2019).

\section{Conclusão}

O conhecimento da epidemiologia das micoses em animais de estimação, principalmente dos dermatófitos, é importante para diminuir a disseminação de infecção por fungos zoofilicos para humanos e auxiliar na obtenção de medidas profiláticas na disseminação da doença entre animais.

O exame direto, utilizado no presente estudo, é uma importante forma de diagnóstico, sendo considerado por Căpitan et al. (2018) como o método mais simples, barato e rápido de diagnóstico, e pode auxiliar o clínico reduzindo o tempo necessário para o início do tratamento, no entanto ainda é pouco sensível, dessa forma faz se necessário o uso da cultura fúngica para obter melhores resultados; ambos, associados à clínica do animal e por vezes outras ferramentas diagnósticas, fornecem um diagnóstico mais preciso para que a terapêutica estabelecida seja eficaz para um melhor tratamento e prognóstico dos casos clínicos.

\section{Referências bibliográficas}

Balda, A. C., Larsson, C. E., Otsuka, M., \& Gambale, W. (2004). Estudo retrospectivo de casuística das dermatofitoses em cães e gatos atendidos no Serviço de Dermatologia da Faculdade de Medicina Veterinária e Zootecnia da Universidade de São Paulo. Acta Scientiae Veterinariae, 32(2), 133-140. DOI: https://doi.org/10.22456/1679-9216.16835

Brilhante, R. S. N., Cavalcante, C. S. P., Soares-Junior, F. A., Cordeiro, R. A., Sidrim, J. J. C., \& Rocha, M. F. G. (2003). High rate of Microsporum canis feline and canine dermatophytoses in Northeast Brazil: epidemiological and diagnostic features. Mycopathologia, 156(4), 303-308. 
DOI: https://doi.org/10.1023/B:MYCO.0000003582.67122.69.

Cabanes, F. J., Abarca, M. L., \& Bragulat, M. R. (1997). Dermatophytes isolated from domestic animals in Barcelona, Spain. Mycopathologia, 137(2), 107-113. DOI: https://doi.org/10.1046/j.1439-0507.2003.00868.x.

Cafarchia, C., Romito, D., Sasanelli, M., Lia, R., Capelli, G., \& Otranto, D. (2004). The epidemiology of canine and feline dermatophytoses in southern Italy. Mycoses, 47(11-12), 508-513. DOI: https://doi.org/10.1111/j.1439-0507.2004.01055.x.

Căpitan, R., Schievano, C., \& Noli, C. (2018). Evaluation of the value of staining hair samples with a modified Wright-Giemsa stain and/or showing illustrated guidelines for the microscopic diagnosis of dermatophytosis in cats. Veterinary Dermatology, 29(4), 308-e106. DOI: https://doi.org/10.1111/vde.12543.

Chaves, L. J. Q. (2007). Dermatomicoses em cães e gatos; Avaliação do diagnostico clínicolaboratorial e dos aspectos epidemiológicos em uma população de portadores de lesões alopécicas circulares. In Departamento de Veterinária: Vol. Master of. Universidade Federal do Ceará.

Coelho, A. C., Alegria, N., \& Rodrigues, J. (2008). Isolamento de dermatófitos em animais domésticos em Vila Real, Portugal. Arquivo Brasileiro de Medicina Veterinária e Zootecnia, 60(4), 1017-1020. DOI: https://doi.org/10.1590/s0102-09352008000400035

Lewis, D. T., Foil, C. S., \& Hosgood, G. (1991). Epidemiology and clinical features of dermatophytosis in dogs and cats at Louisiana State University: 1981-1990. Veterinary Dermatology, 2(2), 53-58. DOI: https://doi.org/10.1111/j.1365-3164.1991.tb00111.x.

Menges, R. W., \& Georg, L. K. (1957). Survey of animal ringworm in the United States. Public Health Reports, 72(6), 503. DOI: https://doi.org/10.2307/4589811.

Miller, W. H., Griffin, C. E., Campbell, K. L., \& Muller, G. H. (2013). Muller and Kirk's Small Animal Dermatology. Elsevier Health Sciences.

Moriello, K. (2019). Dermatophytosis in cats and dogs: a practical guide to diagnosis and treatment. In Practice, 41(4), 138-147. DOI: https://doi.org/10.1136/inp.11539.

Moriello, K. A., Coyner, K., Paterson, S., \& Mignon, B. (2017). Diagnosis and treatment of dermatophytosis in dogs and cats. Clinical Consensus Guidelines of the World Association for Veterinary Dermatology. Veterinary Dermatology, 28(3), 266-e68. DOI: https://doi.org/10.1111/vde.12806.

Neves, R. C. S. M., Cruz, F. A. C. S., Lima, S. R., Torres, M. M., Dutra, V., \& Sousa, V. R. F. (2011). Retrospectiva das dermatofitoses em cães e gatos atendidos no Hospital Veterinário da Universidade Federal de Mato Grosso, nos anos de 2006 a 2008. Ciência Rural, 41(8), 14051410. DOI: https://doi.org/10.1590/S0103-84782011000800017.

Palumbo, M. I. P., Machado, L. H. A., Paes, A. C., Mangia, S. H., \& Motta, R. G. (2010). Estudo epidemiológico das dermatofitoses em cães e gatos atendidos no serviço de dermatologia da Faculdade de Medicina Veterinária e Zootecnia da UNESP-Botucatu. Semina: Ciências Agrárias, 31(2), 459-468. DOI: https://doi.org/10.5433/1679-0359.2010v31n2p459.

Paterson, S. (2010). Manual de doenças da pele do cão e do gato. Guanabara Koogan.

Silva, V. F., Drescher, G., Mattiello, S. P., Kolling, L., Muller, G., Ferronatto, A. I., Santurio, J. M., \& Costa, M. M. (2011). Agentes fúngicos da dermatofitose em cães e gatos do município de Xanxerê, Santa Catarina. Semina: Ciências Agrárias, 32(3), 1095-1100. DOI: https://doi.org/10.5433/1679-0359.2011v32n3p1095

Sparkes, A. H., Gruffydd-Jones, T. J., Shaw, S. E., Wright, A. I., \& Stokes, C. R. (1993). Epidemiological and diagnostic features of canine and feline dermatophytosis in the United Kingdom from 1956 to 1991. The Veterinary Record, 133(3), 57-61. DOI: https://doi.org/10.1136/vr.133.3.57.

Stannard, A. A., \& Pulley, L. T. (1978). Tumors of the skin and soft tissues. In J. E. Moulton (Ed.), Tumors in Domestic Animals (pp. 211-212). University of California Press.

Recebido: 3 de abril, 2020.

Aprovado: 20 de maio, 2020.

Disponível online: 31 julho, 2020.
Licenciamento: Este artigoé publicado na modalidade Acesso Aberto sob a licença Creative Commons Atribuição 4.0 (CC-BY 4.0), a qual permite uso irrestrito, distribuição, reprodução em qualquer meio, desde que o autor e a fonte sejam devidamente creditados. 